\title{
Anatomia Angiográfica do Sistema Venoso Coronário. Aplicações em Eletrofisiologia Clínica
}

\author{
Wesley Duílio S. Melo, Luis A. Renjel Prudencio, Cássia E. Kusnir, A na Lucia N. Pereira, Vera Marques, \\ Magno C. Vieira, Angelo A mato V. de Paola
}

São Paulo, SP

\begin{abstract}
Objetivo - Avaliar as possibilidades diagnósticas e terapêuticas, através de microcateteres introduzidos nas veias do coração, em estudo angiográfico da circulação venosa coronária.

Métodos - Foram realizadas venografias superseletivas do coração em 30 portadores de taquicardia ventricular sustentada, utilizando-se cateter específico que providenciava a oclusão temporária do óstio do seio coronário. A idade média foi de 52,7 (variação de 24 a 76) anos, sendo 25 do sexo masculino e cinco do feminino. As veias foram analisadas de acordo com o número, calibre e distribuição nas paredes anterior e posterior do ventrículo esquerdo (VE).

Resultados - O seio coronário foi cateterizado em todos os pacientes, não havendo desconforto adicional ou complicações em nenhum caso. O número de veias da parede posterior do ventrículo esquerdo foi 3,1 e na parede anterior, 1,9 $\mathrm{p}<0,05$. Calibres das veias coronárias observados: veia interventricular anterior (segmento distal $=1,19 \pm 0,22 \mathrm{~mm}$, segmento médio $=1,65 \pm 0,35 \mathrm{~mm}$ ); veia interventricular posterior (segmento distal $=1,83 \pm 0,47 \mathrm{~mm}$, segmento médio $=$ $2,00 \pm 0,52 \mathrm{~mm}$ ); veia posterior do VE (segmento distal $=$ $1,45 \pm 0,25 \mathrm{~mm}$, segmento médio $=2,49 \pm 0,92 \mathrm{~mm}) ; \mathrm{p}<0,05$.

Conclusão - A técnica de oclusão para o estudo das veias coronárias é exeqüível e segura. O número e o calibre (segmentos médio e distal) das veias da parede posterior do VE são significantemente maiores do que da parede anterior, sugerindo que condições anatômicas para o mapeamento elétrico epicárdico, através das veias coronárias, são mais adequadas na parede posterior do VE.
\end{abstract}

Palavras-chave: angiografia coronária, veia, taquicardia ventricular

\section{Angiography of the Coronary Venous System. Usefulness in Clinical Cardiac Electrophysiology}

Purpose - To study the angiographic anatomy of human coronary veins and the possibility of epicardial venous mapping through microelectrode catheters.

Methods - We evaluated 30 patients with sustained ventricular tachycardia using a catheter which provided occlusion of the coronary sinus ostium during venous angiography. They were 25 males, 5 females, ages ranging from 24 to 76 years (mean $=52.7$ ). The veins were studied according to their number, caliber and distribution in the anterior and posterior wall of the left ventricle.

Results - Coronary sinus was catheterized in all patients. No discomfort or complications were observed. The number of veins from posterior wall of the left ventricle was 3.1 and anterior wall, $1.9, \mathrm{p}<0.05$. The caliber of the coronary veins were: anterior interventricular vein (distal segment $=$ $1.19 \pm 0.22 \mathrm{~mm}$, midle segment $=1.65 \pm 0.35 \mathrm{~mm}$ ), posterior interventricular vein $($ distal segment $=1.83 \pm 0.47 \mathrm{~mm}$, midle segment $=2.00 \pm 0.52 \mathrm{~mm}$ ), left posterior vein (distal segment $=$ $1.45 \pm 0.25 \mathrm{~mm}$, midle segment $=2.49 \pm 0.92 \mathrm{~mm}$ ); $\mathrm{p}<0.05$.

Conclusion - The balloon occlusion technique for coronary venous angiography is feasible and safe. The number and the caliber (distal and midle) of the veins from the posterior wall of the left ventricle were significantly greater than those from the anterior wall. Anatomic conditions for venous epicardial mapping are more adequate in the posterior wall of the left ventricle.

Keywords: coronary angiography, vein, ventricular tachycardia

Arq Bras Cardiol, volume 70 (nº 6), 409-413, 1998

Escola Paulista de Medicina - UNIFESP

Correspondência: Wesley Duílio S. Melo - EPM-UNIFESP - Setor de Eletrofisiologia Clínica - Rua Botucatu, 740 - 04023-062 - São Paulo, SP

Recebido para publicação em 25/11/97

Aceito em 19/3/98
Em 1950, quando surgiram os primeiros relatos da visibilização angiográfica das artérias coronárias ${ }^{1}$, não era possível imaginar os benefícios clínicos que aquelas imagens trariam para o futuro da cardiologia. Sua grande aplicação clínica fez com que, atualmente, essa circulação esteja bem conhecida, havendo poucas dúvidas sobre sua anatomia. 
Embora as veias coronárias estejam anatomicamente bem próximas às artérias, são escassas as publicações que descreveram, morfológica e topograficamente, as veias cardíacas, provavelmente, devido à falta de utilidade clínica que despertasse o interesse pelo estudo dessa circulação.

A maioria das técnicas desenvolvidas para o mapeamento das arritmias consiste na captação de sinais elétricos apenas no endocárdio, limitando a identificação dos focos epicárdicos e mesmo intramiocárdicos. As limitações diagnósticas e terapêuticas dessas técnicas ${ }^{2-6}$ justificaram a utilização de estratégias para o mapeamento epicárdico do coração.

Recentemente, foi demonstrada a possibilidade de mapeamento elétrico epicárdico das arritmias ventriculares com microcateteres, através das veias coronárias por acesso percutâneo ${ }^{7}$. Trata-se de um novo método que necessita de um profundo conhecimento da anatomia da circulação venosa cardíaca, no seu aspecto angiográfico, para delinear as regiões do coração mais favoráveis a este tipo de mapeamento.

A escassez de trabalhos angiográficos descritivos da circulação coronária venosa, sua possibilidade de viabilizar o mapeamento elétrico epicárdico do coração motivaram o presente estudo, objetivando a descrição angiográfica da circulação coronária venosa e a análise dos territórios do coração que permitem a canulação seletiva das veias por microcateteres eletrodos.

\section{Métodos}

Foram estudados, prospectivamente, de fevereiro/95 a outubro/97, 30 portadores de taquicardia ventricular sustentada a serem submetidos a estudo eletrofisiológico para mapeamento e ablação. A idade variou de 24 a 76 (média 52,7) anos; sendo 25 do sexo masculino e cinco do feminino. Treze eram portadores de cardiopatia chagásica crônica, nove com doença arterial coronária e oito de causa idiopática.

A angiografia venosa foi realizada em laboratório de hemodinâmica, sob monitorização contínua com eletrocardiograma de 12 derivações simultâneas, momentos antes de iniciar procedimento principal (estudo eletrofisiológico), com o paciente acordado, em ritmo cardíaco regular (sinusal). O paciente era posicionado em decúbito dorsal, sendo o acesso venoso feito pela veia jugular interna direita com prévia anestesia local (lidocaína $2 \%$ ), posicionando-se introdutores intravasculares 8 French por onde se progredia o cateter angiográfico. A seguir, realizava-se a cateterização do seio coronário sob visão radioscópica com cateter angiográfico 7 French de duas vias (Electro-Catheter Corporation, Raway, NJ, USA) (fig. 1). Uma via desse cateter comunicava-se com sua luz, que exteriorizava-se na sua extremidade distal e era utilizada para injeção de contraste de baixa osmolaridade. A outra via, por onde se insuflava cerca de 1,5cc de ar ambiente, era conectada a um balão situado a $1 \mathrm{~cm}$ da extremidade distal do cateter. Esse sistema possibilitava a oclusão temporária do seio coronário próximo ao seu óstio ao se insuflar o balão nesse local, impedindo que o

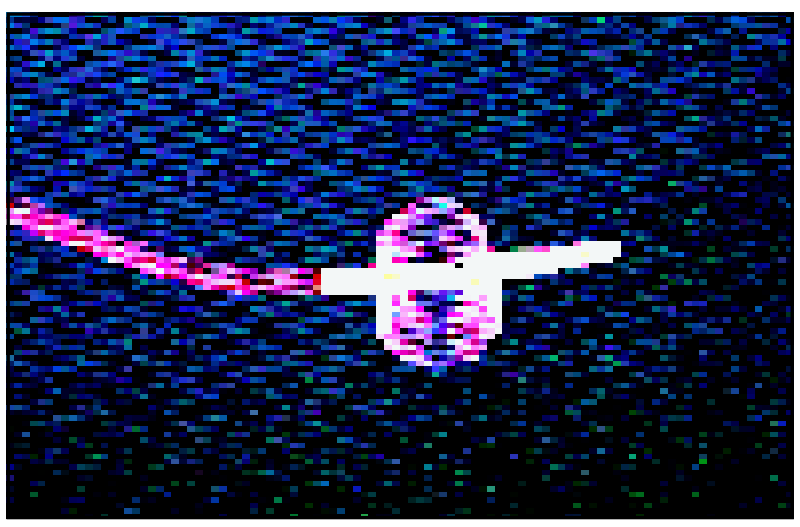

Fig. 1 - Cateter angiográfico utilizado neste estudo. Detalhes no texto.

sangue proveniente do retorno venoso cardíaco fosse drenado no átrio direito (AD) (fig. 2).

Durante o estudo angiográfico foram obtidas imagens em projeções oblíquas anterior direita $\left(30^{\circ}\right.$ ou $\left.45^{\circ}\right)$ e esquerda $\left(30^{\circ}\right.$ ou $\left.45^{\circ}\right)$.

O calibre das veias interventricular posterior, interventricular anterior e posterior do ventrículo esquerdo (VE) foi avaliado comparativamente por segmentos (proximal, médio e distal). A distribuição venosa foi avaliada de acordo com sua disposição nas paredes anterior e posterior do VE.

A análise angiográfica foi executada, em data posterior à realização do exame, através do método de angiografia
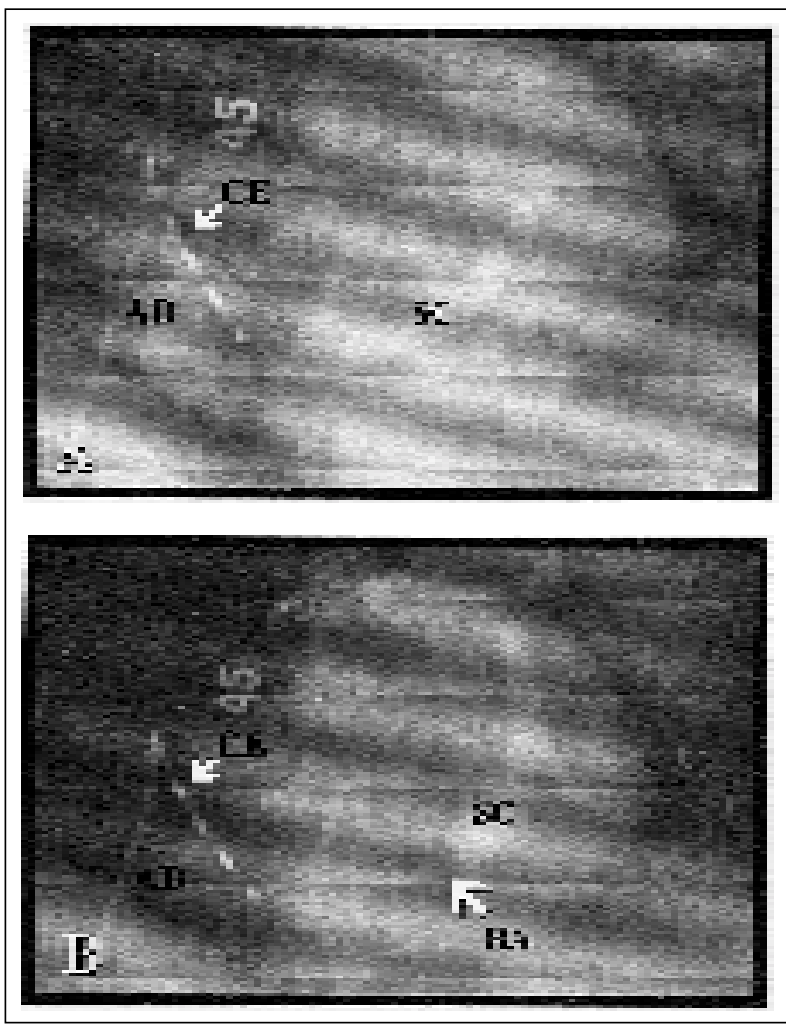

Fig. 2 - Angiografia do seio coronário em incidência oblíqua anterior esquerda a $45^{\circ}$ : A) refluxo de constraste para o interior da câmara atrial direita; B) com o balão insuflado, o contraste permanece no interior do seio coronário, possibilitando a visualização de seus ramos. $\mathrm{AD}$ - átrio direito; $\mathrm{SC}$ - seio coronário; $\mathrm{CE}$ - cateter eletrodo posicionado em ventrículo direito; BA- balão. 
coronária quantitativa: Cardiovascular Measurement System, version 3.0 - QCA/CMS - (Medical Imaging Systems, Neunen, The Netherlands) acoplada a um cine projetor Tagarno ${ }^{\circledast}$. Esse sistema foi desenvolvido, originalmente, para análise do calibre de artérias coronárias, e foi por nós adaptado, para o estudo da circulação venosa coronária, face à inexistência de um software específico.

A imagem a ser analisada era selecionada e, em seguida, realizada a calibração, utilizando-se, para isso, um cateter de calibre conhecido com contraste em seu interior. Concluídas estas etapas, procedia-se à mensuração do segmento escolhido, por meio da detecção automática dos contornos venosos.

Para a análise estastística foram aplicados os testes de Wilcoxon, de Tukey e análise de variância. Foi considerado significante valores de $\mathrm{p}<0,05$.

\section{Resultados}

Não houve dificuldade técnica na canulação do óstio do seio coronário com o cateter angiográfico, obtendo-se boa definição angiográfica de suas tributárias em quase todos os casos.

Durante o estudo angiográfico, não houve desconforto para o paciente, não se detectando nenhuma alteração eletrocardiográfica e/ou hemodinâmica.

O seio coronário apresentou angiografia normal em todos os 30 pacientes estudados. O diâmetro médio luminal ao nível de seu óstio foi de $17,01 \mathrm{~mm}$.

A pequena veia cardíaca, detectada em um caso, originou-se no bordo direito, percorrendo, posteriormente, $\mathrm{o}$ sulco atrioventricular direito e drenando no seio coronário.

A veia de Marshall, detectada em oito casos, originouse na face póstero-superior do átrio esquerdo, ao nível da veia pulmonar superior esquerda, dirigindo-se para baixo, drenando no bordo superior do seio coronário.

A veia interventricular anterior, visualizada em todos os pacientes, tem sua origem próxima ao ápice cardíaco na sua face anterior, ascendendo pelo sulco interventricular anterior e, ao se aproximar da bifurcação do tronco principal

\begin{tabular}{|lccc|}
\hline \multicolumn{4}{|c|}{ Tabela 1 - Calibre das veias coronárias do ventrículo esquerdo } \\
\hline & Segmento proximal & Segmento médio & Segmento distal \\
\hline IVA & $2,98 \pm 0,72$ & $1,65 \pm 0,35$ & $1,19 \pm 0,22$ \\
IVP & $3,44 \pm 1,11$ & $2,00 \pm 0,52^{*}$ & $1,83 \pm 0,47 *$ \\
VP & $3,35 \pm 0,94$ & $2,49 \pm 0,92^{*}$ & $1,45 \pm 0,25^{*}$ \\
\hline \multirow{2}{*}{$*$ p $<0,05$. IVA=veia interventricular anterior, IVP=veia interventricular pos- } \\
terior, VP=veia posterior do ventriculo esquerdo \\
\hline
\end{tabular}

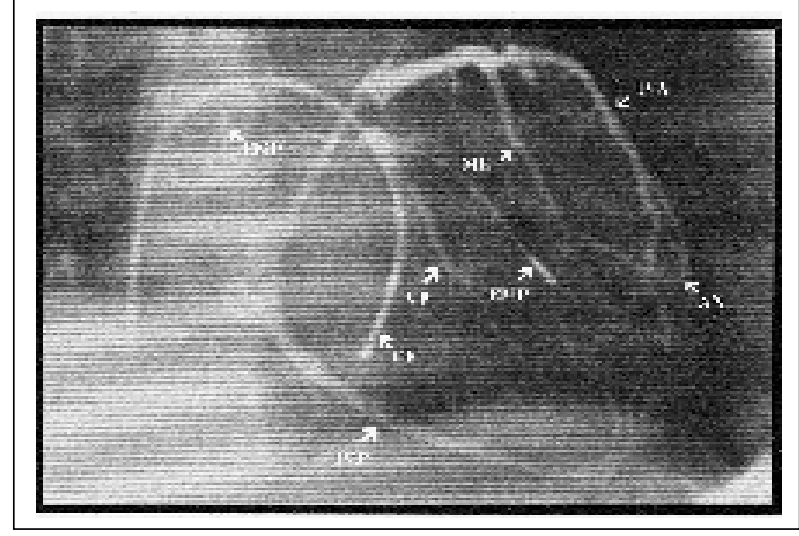

Fig. 3 - Angiografia venosa coronária em incidência oblíqua anterior direita, demonstrando alguns ramos do seio coronário. IVA- veia interventricular anterior; ME- veia marginal esquerda; IVP- veia interventricular posterior; CE- cateter eletrodo posicionado em ventrículo direto; EMP- eletrodos de marcapasso definitivo; $\mathrm{AN}$ - anastomose entre a veias IVA e ME

da artéria coronária descendente anterior, dirigiu-se para a esquerda, circundando, posteriormente, o sulco atrioventricular esquerdo, passando a se denominar seio coronário (fig. 3).

A veia interventricular posterior, também visibilizada em todos os pacientes, originou-se no ápice cardíaco na sua face posterior, ascendendo pelo sulco interventricular posterior, e drenando no seio coronário (fig. 3).

As veias posteriores do $\mathrm{VE}$ apresentam-se em número variável. Originavam-se próximo do bordo esquerdo, dirigindo-se superior e paralelamente à veia interventricular posterior, atravessando a porção média da face posterior do VE para terminarem no bordo inferior do seio coronário. A veia posterior do VE foi observada em 29 casos, apresentando-se em número variável de um a quatro (mediana $=2$ ) e, geralmente, representada por uma veia bastante desenvolvida em relação às demais posteriores do VE (fig. 3).

A veia marginal observada em 11 pacientes, originouse no bordo esquerdo, dirigiu-se superiormente drenando no bordo inferior do seio coronário (fig. 3).

Foi demonstrado que os calibres dos segmentos médio e distal das veias interventricular posterior e posterior do VE, são maiores que o da veia interventricular anterior (tab. I). Verificou-se que a veia interventricular anterior apresentou um decréscimo mais intenso no seu calibre em relação às demais, à medida em que avançava para o território distal (gráfico 1).

A despeito dessas veias drenarem no seio coronário, para essa análise foi considerada como segmento proximal aquele mais próximo ao seio coronário e, segmento distal, o mais distante.

\begin{tabular}{|lccccccc|}
\hline \multicolumn{7}{|c|}{ Tabela 2 } & - Anastomoses angiográficas das veias coronárias \\
\hline Anastomoses & IVA-IVP & IVP-VP & VP-VP & IVA-VP & IVP-ME & VP-ME & IVA-ME \\
\hline Número & 29 & 18 & 14 & 08 & 07 & 03 & 02 \\
Calibre & $1,41 \pm 0,31$ & $1,28 \pm 0,34$ & $1,27 \pm 38$ & $1,03 \pm 0,37$ & $1,34 \pm 0,30$ & $1,16 \pm 0,16$ & $0,93 \pm 0,13$ \\
\hline IVA=veia interventricular anterior, IVP=veia interventricular & posterior, ME=veia marginal esquerda, VP=veia posterior do ventriculo esquerdo. \\
\hline
\end{tabular}




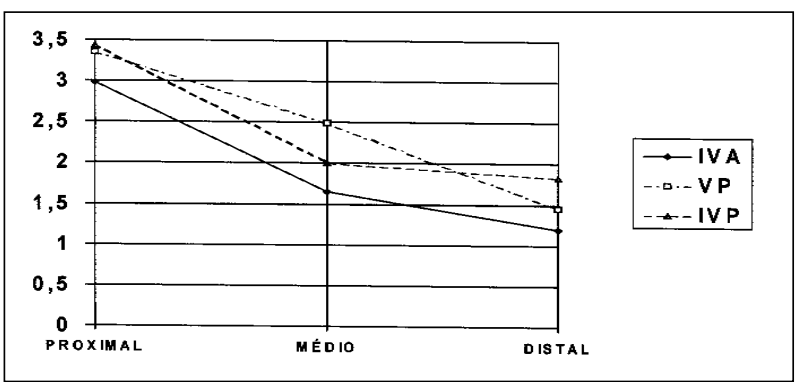

Gráfico 1 - Representação esquemática demonstrando maior estreitamento da veia interventricular anterior (IVA) quando comparada com as veias interventricular posterior (IVP) e posterior do ventrículo esquerdo (VP)

Foi analisado o número de veias com calibre superior a $1,3 \mathrm{~mm}$, nas paredes anterior e posterior do VE. Esse valor $(1,3 \mathrm{~mm})$ foi utilizado por ser considerado o diâmetro mínimo necessário para a adequada manipulação do cateter mapeador intracoronário, cujo diâmetroé 3,3 French $(1,1 \mathrm{~mm})$. A parede posterior do $\mathrm{VE}$ apresentou um número médio de veias de 3,3, valor significantemente maior, quando comparado com os obtidos da parede anterior: $1,8, \mathrm{p}<0,05$.

As anastomoses estiveram presentes em grande número, constituindo uma particularidade, freqüentemente não encontrada na circulação arterial. A análise das comunicações, envolvendo as tributárias do seio coronário, encontra-se na tabela II.

\section{Discussão}

Apesar dos avanços na área médica envolverem requintes tecnológicos e mecanismos muito refinados, a abordagem de variáveis relacionadas às ciências básicas trazem freqüentemente contribuições significantes. O conhecimento da anatomia arterial do corpo humano e, especialmente, a do coração é absolutamente imprescindível para o tratamento de várias doenças.

A despeito da inexistência de múltiplas aplicações clínicas imediatas, acreditamos que o conhecimento minucioso da anatomia angiográfica das veias coronárias em seres humanos, que se inicia com esse estudo, contribuirá para o

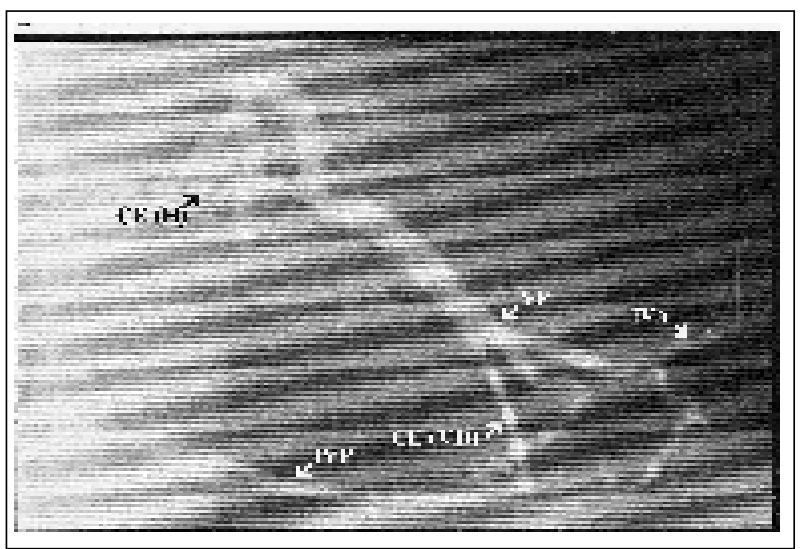

Fig. 4 - Angiografia venosa coronária em incidência oblíqua anterior direita, demonstrando anastomoses entre as veias: interventricular anterior (IVA), posterior do ventrículo esquerdo (VP), e interventricular posterior (IVP). CE- cateteres eletrodos posicionados ao nível do feixe de His (H) e ventrículo direito (VD).

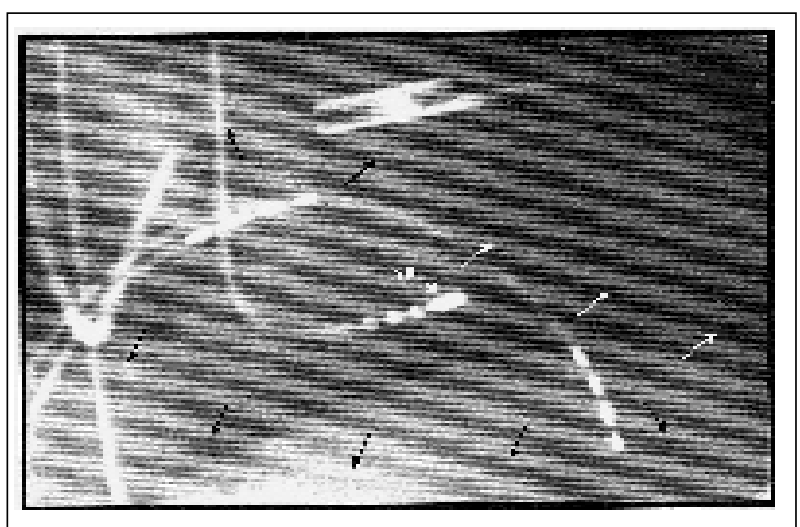

Fig. 5 - Mesmo caso da figura 4. Mapeamento elétrico epicárdico da parede posterior do ventrículo esquerdo (VE) por meio de um único microeletrodo multipolar (setas), posicionado nas veias posterior do VE e interventricular posterior através de uma anastomose. ABL- cateter de ablação posicionado no VE.

aparecimento de novas possibilidades diagnósticas e terapêuticas.

O aspecto angiográfico da rede venosa do coração foi pouco estudado, limitando-se, em alguns trabalhos, a análise morfológica do seio coronário, sem analisar especificamente a suas tributárias ${ }^{1,8-11}$.

Constatamos em estudo piloto que, ao injetarmos contraste no seio coronário, houve rápida dispersão do mesmo para o AD. O fato de a injeção de contraste ser realizada contra o fluxo sangüíneo venoso do coração, não permitiu a observação de nenhum ramo do seio coronário. Esse problema técnico fundamental só foi contornado quando foi utilizado um cateter elaborado especificamente para esse fim $^{12}$, possibilitando a adequada opacificação desses vasos e fornecendo detalhes impossíveis de serem registrados com cateteres angiográficos tradicionais.

Face a inexistência de software específico para a circulação coronária venosa, adaptamos a análise angiográfica realizada por intermédio do método QCA/CMS, não havendo necessidade de alterações em sua estrutura básica o que poderia comprometer os resultados. Acreditamos que sua utilização foi de suma importância, sendo possível obter dados mais precisos das variáveis analisadas.

Apesar de estudos, utilizando ou não marcadores radioativos e esferas de vidro, descreverem a presença de comunicações com um calibre entre 70 a 180 micra na árvore arterial coronária ${ }^{13,14}$, anastomoses angiográficas são raramente visualizadas na ausência de obstruções coronárias significantes.

No nosso estudo, onde não foram constatadas lesões nas veias coronárias, observou-se um grande número de anastomoses entre as veias cardíacas superficiais, principalmente, as que envolviam as veias posteriores do VE e ainda a presença, na quase totalidade dos casos, de uma anastomose entre a veia interventricular anterior e veia interventricular posterior (fig. 4).

A presença desta rica rede anastomótica pode eventualmente facilitar o mapeamento epicárdico de determinadas áreas com a utilização de apenas um microeletrodo (fig. 5).

Alguns autores baseados em estudos experimentais e anatomopatológicos descreveram que o desenvolvimento 
de aterosclerose está relacionado ao fluxo turbulento encontrado em algumas artérias ${ }^{15,16}$. Acreditamos, portanto, que o fato da circulação venosa coronária estar em um sistema de baixas pressões explicaria a ausência de lesões obstrutivas em nosso material.

A visualização da pequena veia cardíaca em apenas um paciente pode ser explicada pela sua posição anatômica, dificultando sua cateterização seletiva. O pequeno calibre da veia de Marshall e a provável presença de uma válvula ostial no ponto de sua drenagem no seio coronário pode justificar sua visualização em somente oito casos.

O maior número e calibre das veias da parede posterior do VE e o "afunilamento" menos intenso destas mesmas veias, implicam em um acesso mais fácil dos microcateteres, facilitando o mapeamento venoso epicárdico nesta área, quando comparado com a parede anterior.

O desenvolvimento de novos cateteres com menor perfil e maior flexibilidade deverão facilitar o mapeamento de áreas com veias coronárias menos calibrosas.

A ablação por cateter de taquicardia ventricular é tecnicamente desafiante. O mapeamento epicárdico intravascular realizado através das veias coronárias é exequiível e pode ser de grande auxilio para a localização de focos de taquiarritmias ventriculares no laboratório de eletrofisiologia. Finalmente, constatamos que a angiografia do sistema venoso coronário é uma técnica segura, com boa definição angiográfica das veias coronárias, não havendo complicações em nenhum caso estudado.

\section{Referências}

1. Gordon AJ, Brahms SA, Sussman ML - Visualization of the coronary circulation during angiocardiography. Am Heart J 1950; 39: 114-24.

2. Fontaine G, Tonet Jl, Frank R, Rougier I - Clinical experience with fulguration and antiarrhythmic therapy for the treatment of ventricular tachycardia. Chest, 1989; 95: 785-97.

3. Morady F, Sheinman MM, Di Carlo Jr LA et al - Catheter ablation of ventricular tachycardia with intracardiac shocks: results in 33 patients. Circulation 1987; 75(5): 1037-49.

4. De Paola AAV, TÁvora MZP, Mendonça A, GondimFAA, Hara VM, MartinezEE - Ablação por cateter de taquicardia ventricular sustentada, em pacientes com cardiopatia chagásica crônica, com a utilização de radiofrequência. Reblampa 1994; 7(3): 158 (Resumo)

5. Morady F, Harvey M, Kalbfleisch SJ, El-Atassi R, Calkins H, Langberg JJ Radiofrequency catheter ablation of ventricular tachycardia in patients with coronary artery disease. Circulation 1993; 87(2): 363-72.

6. Gonska BD, Brune S, Bethge KP, Kreuzer H - Radiofrequency catheter ablation in recurrent ventricular tachycardia. Eur Heart J 1991; 12: 1257-65.

7. De Paola AAV, Melo WDS, Távora MZ, Martinez EE - Coronary venous mapping in patients with sustained ventricular tachycardia. J. Am Coll Cardiol 1997; 29(2-A): 202A.

8. Tori G - Radiological visualization of the coronary sinus and coronary veins. Acta Radiol 1952; 36: 405-10.

9. Gensini GG, Di Giorgi S, Coskun O, Palacio A, Kelly AE - Anatomy of the coronary circulation in living man. Circulation $1965 ; 31$ : 778-84.

10. Doig JC, Saito J, Harris L, Downar E - Coronary sinus morphology in patients with atrioventricular junctional reentry taquycardia and other supraventricular tachyarrhythmias. Circulation 1995; 92: 436-41.

11. Chiang C, Chen S, Yang C et al - Major coronary sinus abnormalities: identification of occurrence and significance in radiofrequency ablation of supraventricular tachycardia. Am Heart J 1994; 127(5): 1279-89.

12. Arruda $\mathrm{M}$, Otomo $\mathrm{K}$, Tondo $\mathrm{C}$ et al - Coronary sinus angiography using an "occlusion" technique as an aid to RF ablation of epicardial accessory pathways. PACE 1995; 18: 833 (abstract).

13. Blumgart HL, Schlesinger MJ, Davis D - Studies on the relation of the clinical manifestations of angina pectoris, coronary thrombosis, and myocardial infarction to the pathologic findings. Am Heart J 1940; 19(1): 1-91.

14. Prinzmetal M, Simkin B, Bergman HC, Kruger HE-Studies on the coronary circulation. II. The collateral circulation of the normal human heart by coronary perfusion with radioactive erythrocytes and glass spheres. Am Heart J 1947; 33 : 420-42.

15. Davies PF, Remuzzi A, Gordon EJ, Dewey JR CF, Gimbrone JR MA - Turbulent fluid shear stress induces vascular endothelial cell turnover in vitro. Proc Natl Acad Sci 1986; 83: 2114-7.

16. Glagov S, Ozoa AK - Significance of the relatively low incidence of atherosclerosis in the pulmonary, renal and mesenteric arteries. Ann NY Acad Sci 1968; 149: $940-55$. 